\title{
SECULAR CHANGES IN ANTHROPOMETRIC MEASUREMENTS OF SCHOOLCHILDREN IN ANKARA, TURKEY (1950-2017)
}

\author{
Başak Koca Özer, Ayşegül Özdemir \\ Department of Anthropology, Faculty of Languages, History and Geography, \\ Ankara University, Ankara, Turkey
}

\begin{abstract}
This study aimed to examine the physical growth of Turkish children and determine secular changes in their height and other anthropometric measurements since 1950. For this purpose, three cross-sectional surveys of a total of 4,902 healthy schoolchildren from Ankara (2,490 boys and 2,412 girls) between the ages of 6 and 17 years conducted in 1950, 2005 and 2017 were studied. Body height and sitting height were measured, centile curves were constructed using the LMS method, and sitting height/height ratio was calculated. The results of this study demonstrated positive secular changes in height and sitting height. The increasing rate of height for boys was approximately $2.5 \mathrm{~cm} /$ decade for their respective pubertal ages, whereas for girls, the rate was approximately $1.9 \mathrm{~cm} /$ decade at ages 9,10 and 12 ; however, at the age of 11 , the increment in girls was over $2 \mathrm{~cm} /$ decade. Data sets presented sexual differences at most ages, arising due to a difference in response to environmental and socio-economic conditions. However, due to the socio-economic fluctuations in Turkey, secular increase was not comparable to the increase experienced within developed countries; therefore a continuation of this secular increase can be expected in the near future.
\end{abstract}

Keywords: secular change; anthropometrics; height; schoolchildren; Turkey

\section{INTRODUCTION}

Growth can be a good determinant of public health and indicates the effect of environmental conditions on human growth over long periods of time. Furthermore, it clarifies physiological inter-generational relationships $[4,13,24$, 
$31,33,35,37]$. Although the initial determinants of secular change of growth are generally considered to be greater availability of public health services and improved nutrition, other factors have been suggested by various authors. These include cumulative socio-economic circumstances such as migration, hygienic experience, sanitation, education, income and reduction of child labor $[4,23,37]$. This objective phenomenon has been well-documented over the last two centuries in many industrialized countries around the world $[2,4,16$, $25,34]$.

There is a lack of a continuous growth-monitoring programme providing data on children and adolescents in Turkey. It should be noted that, until the late 1930s, no information was available on anthropological investigation of Turkish schoolchildren's physical growth status [19]. Although two studies by Alantar [1] and Tümay [36] were published at the first Turkish Paediatrics Congress, they had methodological limitations in terms of data analysis and sample selection. Kansu [19] published data on the anthropometric measurements of children and adolescents. This study also included other cross-sectional studies conducted at the same time by Çınar [14], Gökçül [18] and Kinay [20]. Pioneering studies in this field were conducted between the 1940 s and the 1960s to determine local standards for growth and development in different parts of the country. In the 1970s, the first growth standards for schoolchildren were developed in Istanbul. Although in 1988 and 1992, a larger study was conducted $[15,32]$, in these studies, only the percentile curves of children of a higher social status were developed, thus referring to higher socio-economic strata (SES) [21,22].

The purpose of the present study is to determine up-to-date anthropometric measurements of Turkish school children and adolescents as well as secular changes in Turkey over the last seven decades.

\section{MATERIAL AND METHODS}

The dataset of this study includes three cross-sectional anthropometric surveys that include 4,902 healthy schoolchildren (2,490 boys and 2,412 girls). These surveys were consecutively conducted in 1950, 2005 and 2017 in Ankara, the capital of Turkey. The 1950 anthropometric survey was conducted between March and May 1950 on 1990 children (1020 boys and 970 girls) between the ages of 8 and 17 years. The data was collected from children attending primary, secondary and high schools $[6,7,8]$. Bostanc1 subsequently published data on children between the ages of 9 and 16 year, and this is the first time 
that the complete data set of the 1950 anthropometric survey is presented here with new statistical approaches. The 2005 anthropometric survey was conducted between November and December on 1,427 schoolchildren (709 boys and 718 girls) between the ages of 6 and 17 years [21]. The data was collected from children attending private and public primary and high schools. The 2017 anthropometric survey was conducted between March and May on 1485 schoolchildren ( 761 boys and 724 girls) aged 6-17 years. This survey was conducted with the permission of the Turkish Ministry of Education and local area boards of education and, ethical approval was received from Ankara University Ethical Commission [171-1227, 02.07.2014], and consent was taken from each child's parents. The data was collected from public elementary, secondary and high-school children.

Height and sitting height were measured to the nearest mm using a Martin type anthropometer $[28,29,38]$ while the subjects were wearing light clothes without shoes by a single trained team in each survey. Following this, the sitting height / height ratio was calculated. Centile curves were constructed by using the LMS method $[12,13]$. Sex differences were analysed with the t-test using the SPSS 20.0 programme.

\section{RESULTS}

Sample size, mean, standard deviation, L, M, S values and centiles are given in Tables 1-4 for each survey year. The results showed that sexual dimorphism was greater in earlier studies (1950 and 2005) at early ages ( $p<0.001)$. The increase in height as a function of age also showed an early growth spurt in girls in recent surveys. Sexual dimorphism was significant in terms of height in each successive survey year. The final height difference between sexes was determined as follows in the respective surveys: $7 \mathrm{~cm}$ in 1950, $13 \mathrm{~cm}$ in 2005, and $14 \mathrm{~cm}$ in 2017 . The results indicated that since 1950, secular increase in terms of height was prominent in both sexes, but it was greater for boys. In the 2005 and 2017 surveys, the height increase for the $97^{\text {th }}$ percentile was more pronounced (Tables 1 and 2).

Results showed similar growth patterns for sitting height, indicating significant secular increases. It should be noted that the increment in sitting height increased with each survey year. Moreover, sexual dimorphism was evident in these three surveys ( $\mathrm{p}<0.05 ; \mathrm{p}<0.001$ ) (Tables 3 and 4 ). Study results revealed that, although there is an increase in sitting height, the increment in height is also prominent due to an increment in leg length. 


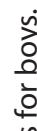

co

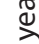

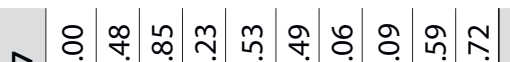

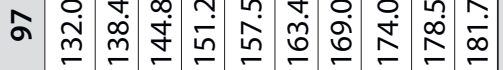

ก ᄂ

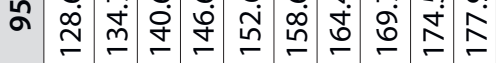

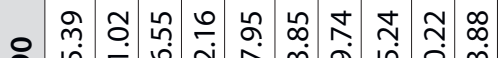

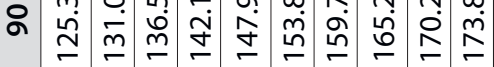

ก

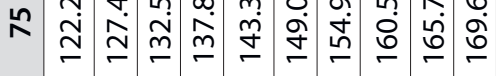

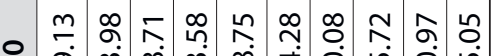

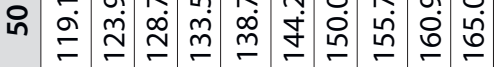

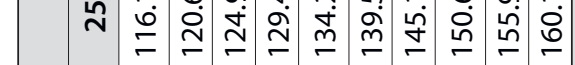

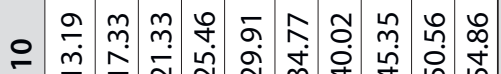

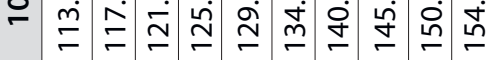

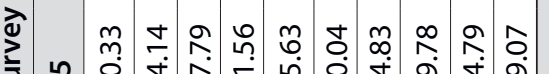

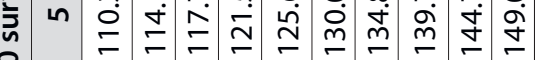

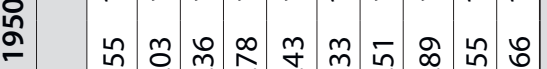

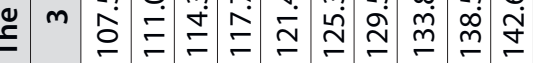

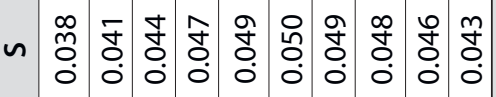

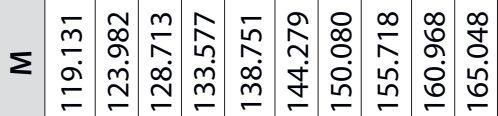

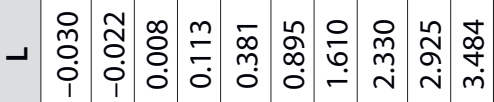

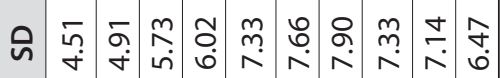

응

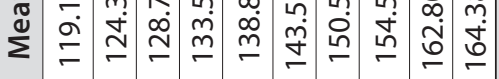

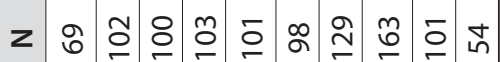

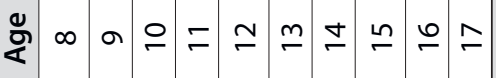

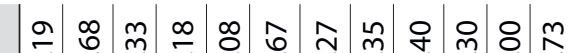

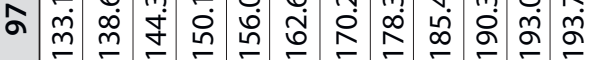

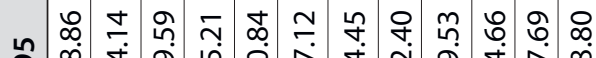

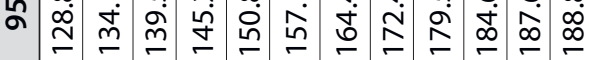
舟

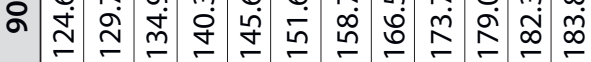

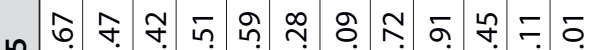

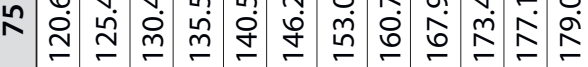
-

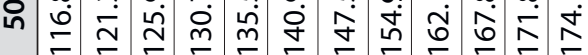
$\infty$ m

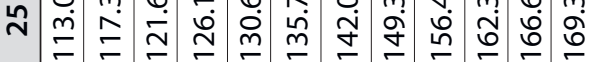
은 굴

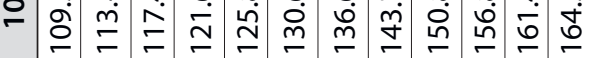
ঐ) น

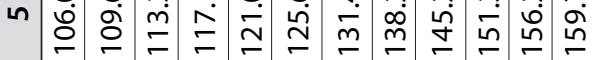
N $\delta$ m

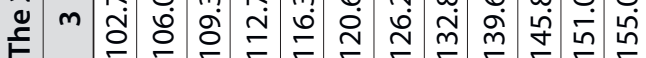

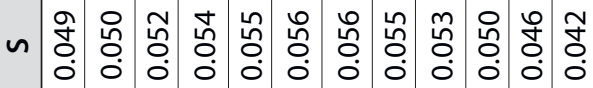
ఫ $\Sigma$ 我

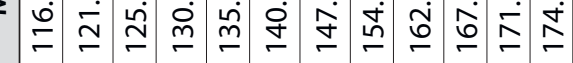

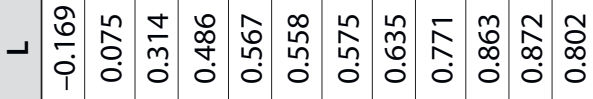

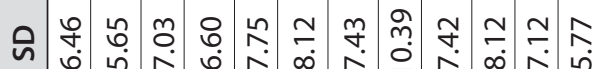

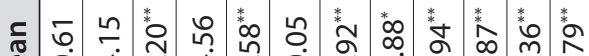

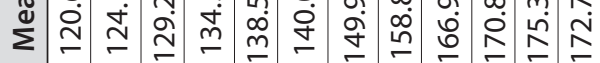

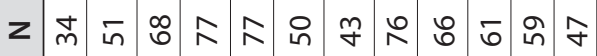

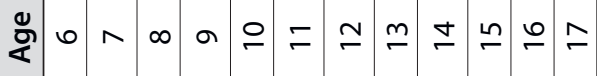




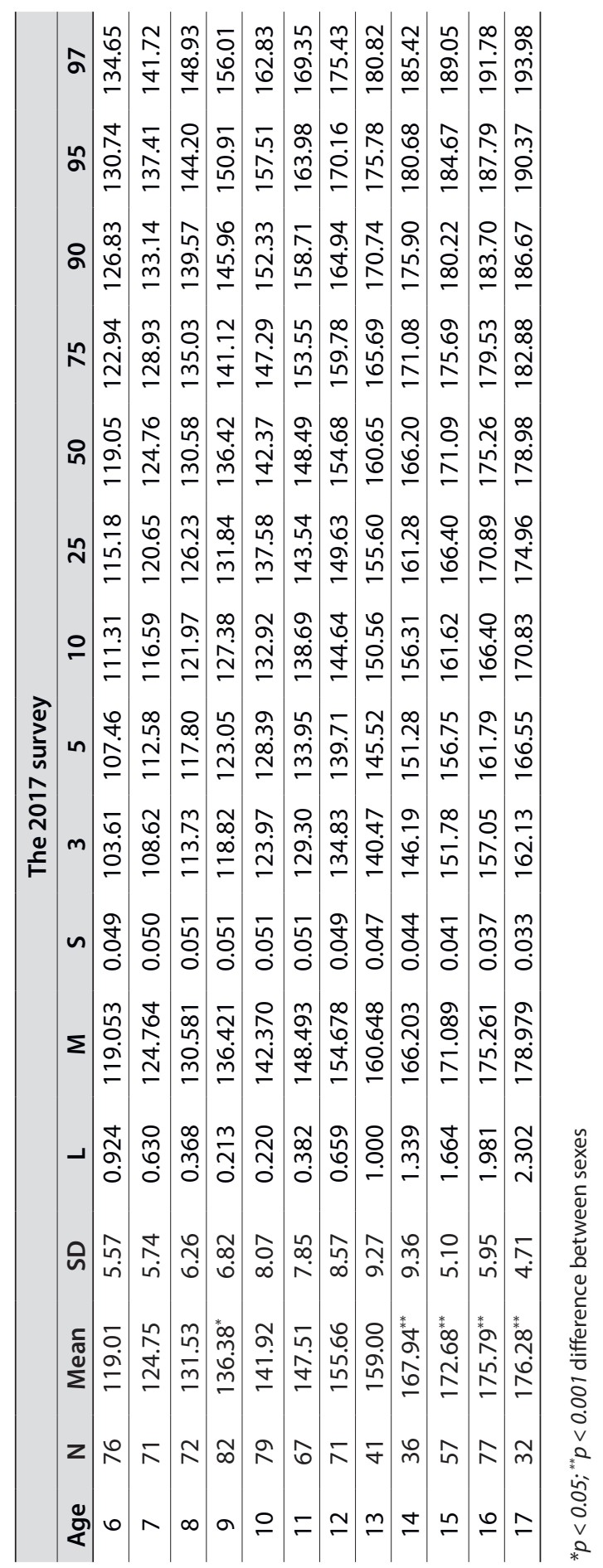


홓

ปั

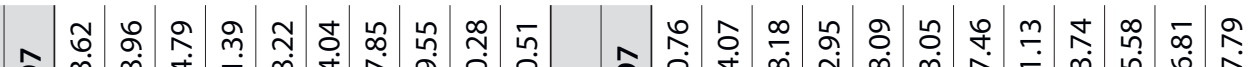

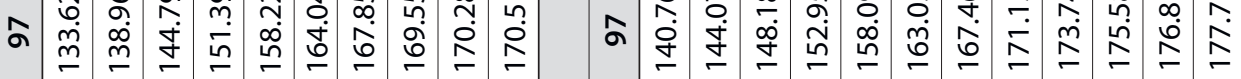

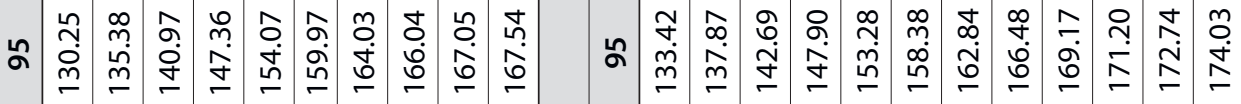

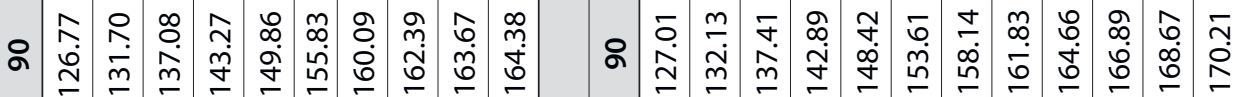
ผ 드 으 우

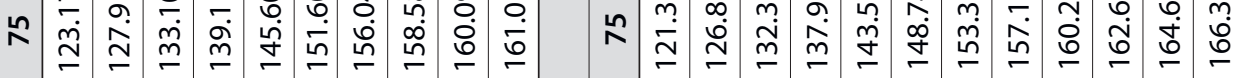

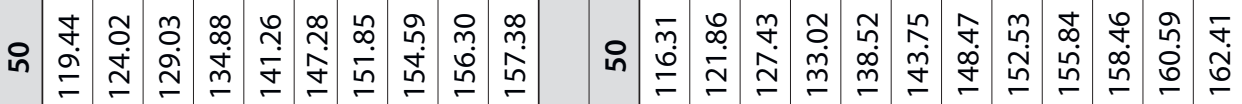
n

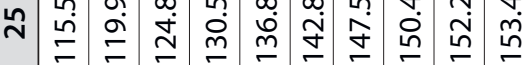

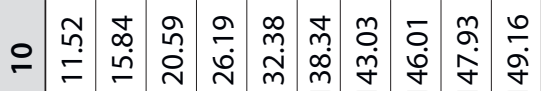

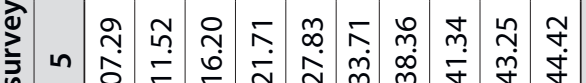

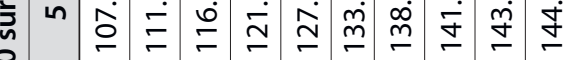

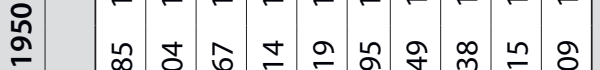
I $m$ 으

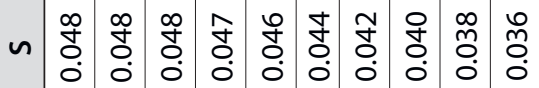

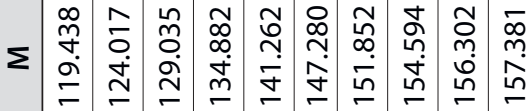

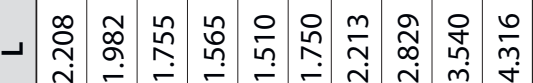

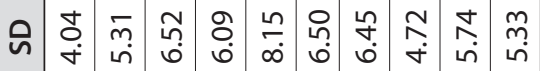

๕

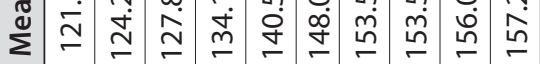

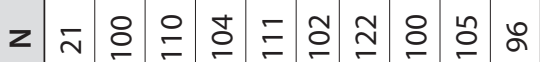

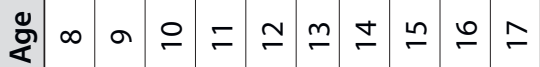

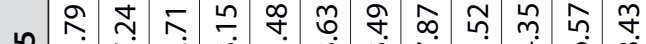

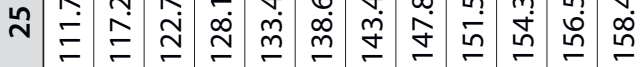

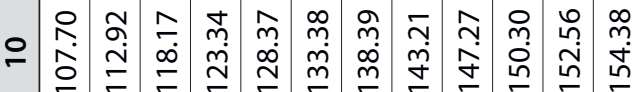

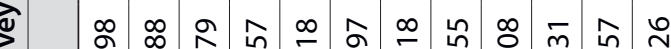

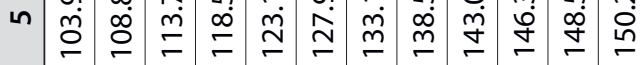
กิ 吕

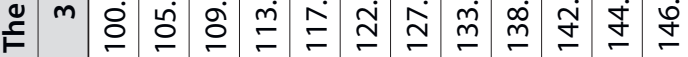

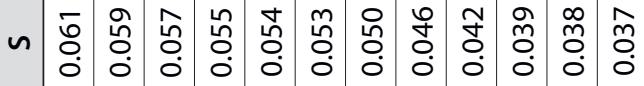

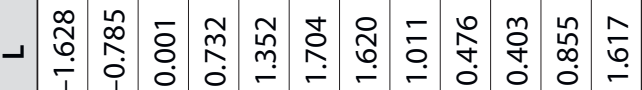

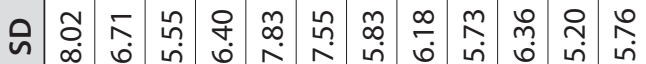
๘

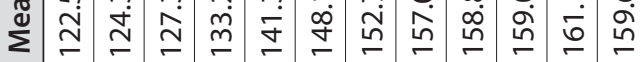
z

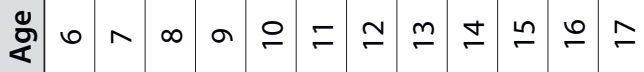

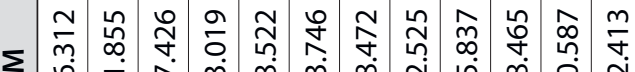

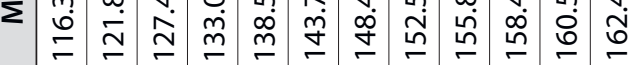




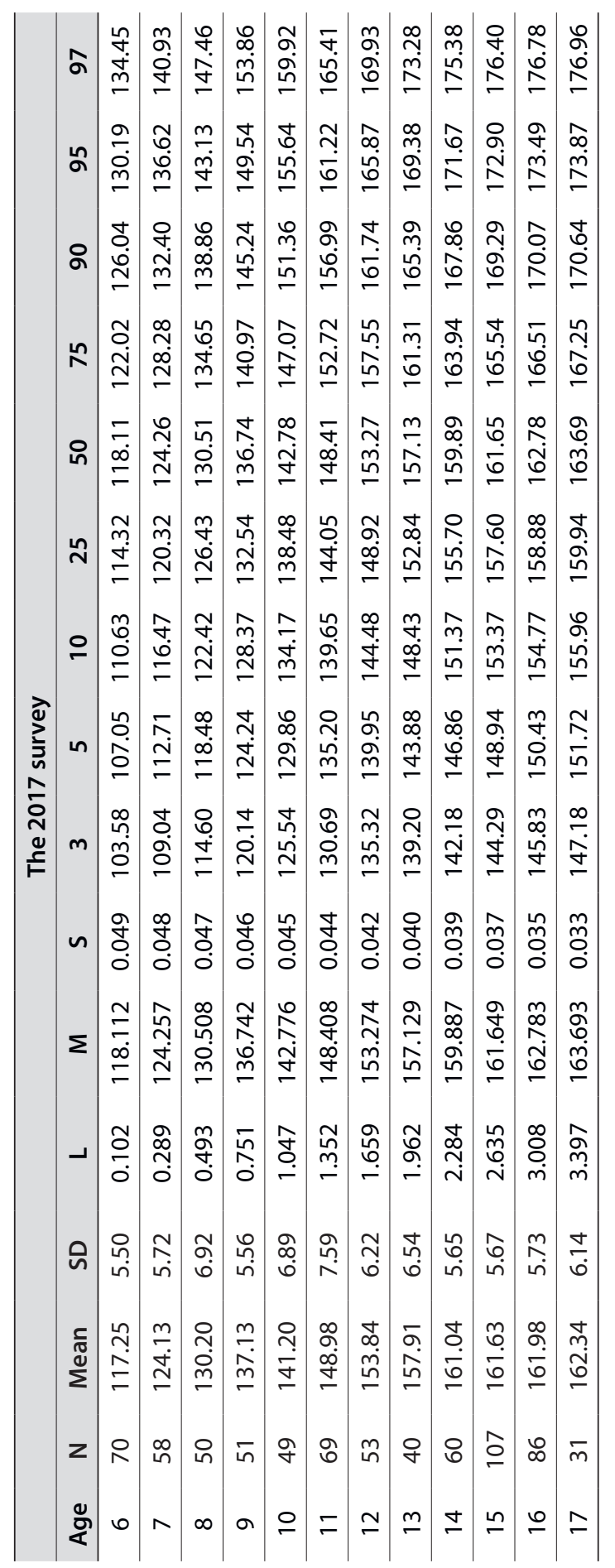


م|

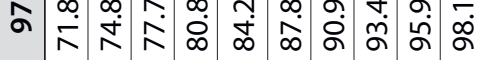

ก นก กุ

んน

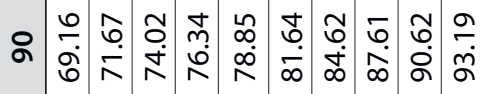

n

N $\begin{aligned} & n \\ & \end{aligned}$

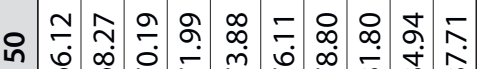

in

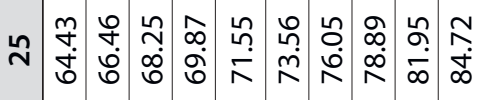

으 일

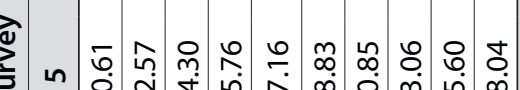

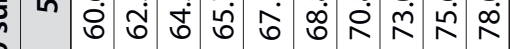

을 ₹

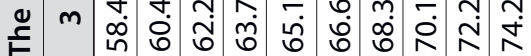

$\sum$

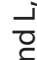

\%

$\frac{\tilde{n}}{\frac{\pi}{\sigma}}$

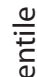

$\checkmark$

$\frac{\frac{\pi}{5}}{\frac{5}{2}}$

$\stackrel{5}{2}$

ֻัฐ

है

$\frac{\sqrt{2}}{\frac{0}{4}}$

$\stackrel{\text { D }}{\text { 证 }}$

$\stackrel{\dot{m}}{\frac{0}{0}}$

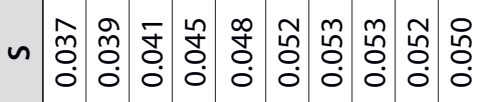

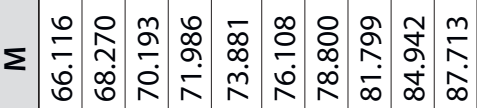

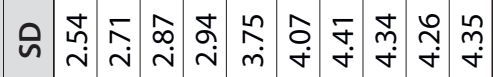

ร

$\sum$ 幽

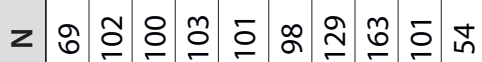

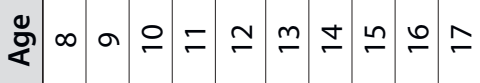

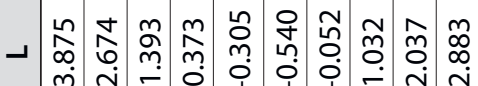

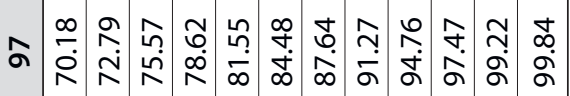

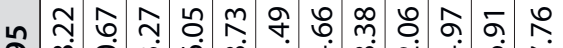

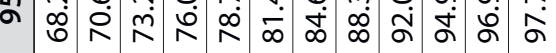

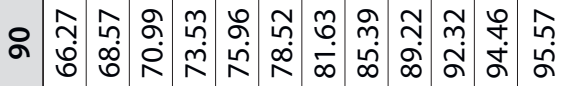

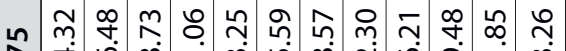

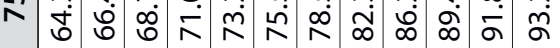

-

ผ

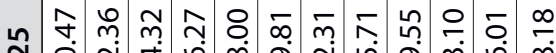

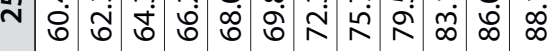

- นำ

-

\ঠ 败

ㅊำ

$m$ 苟

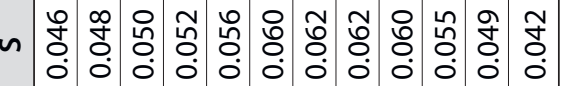

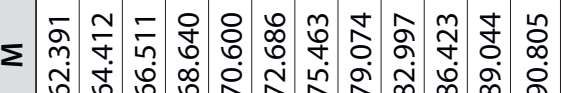

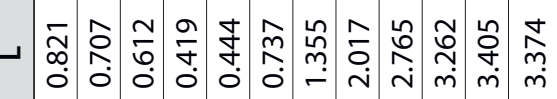

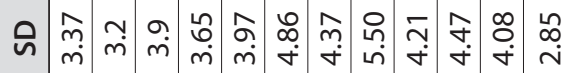

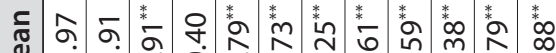

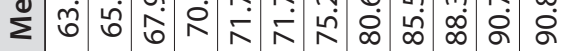

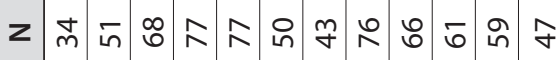

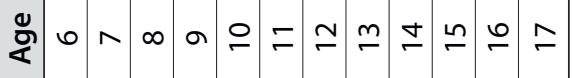




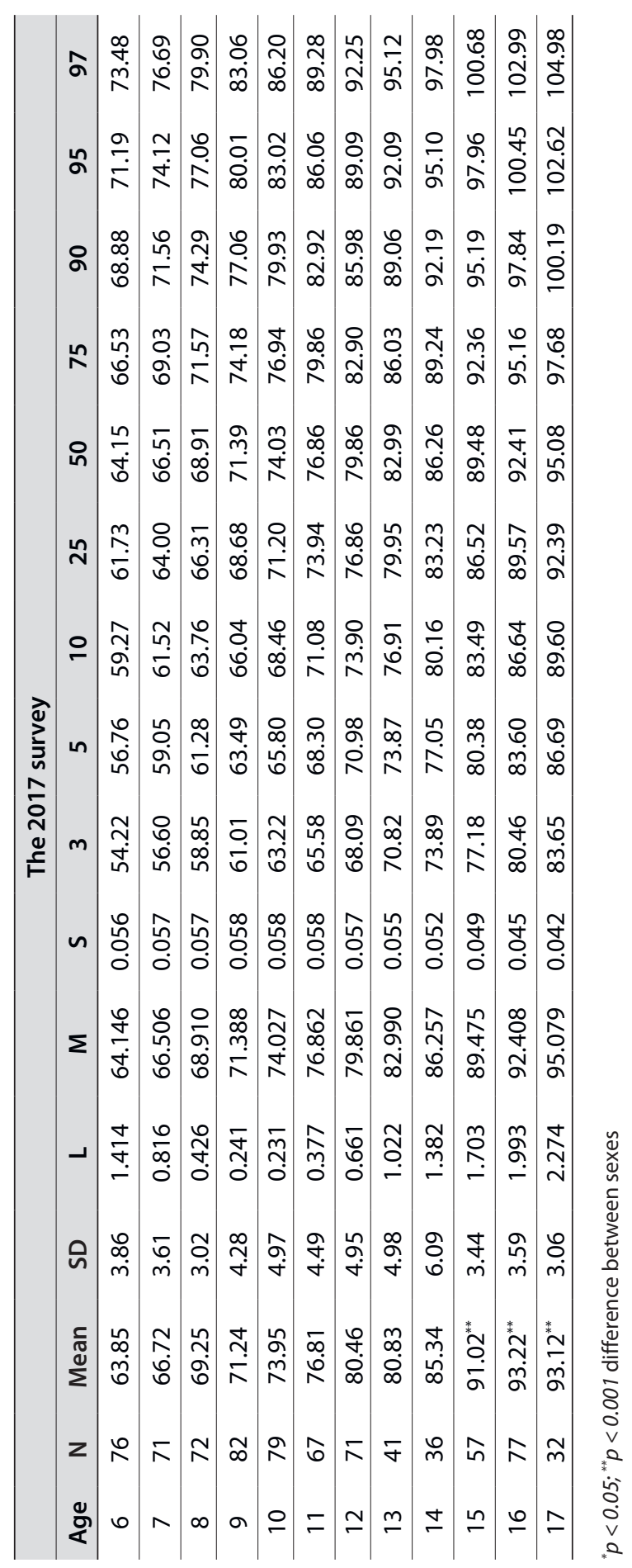




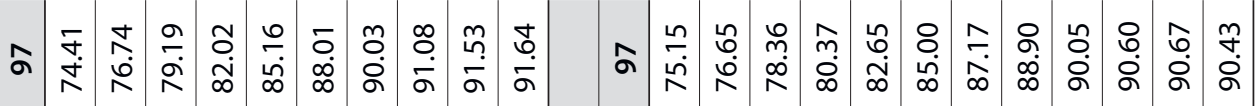

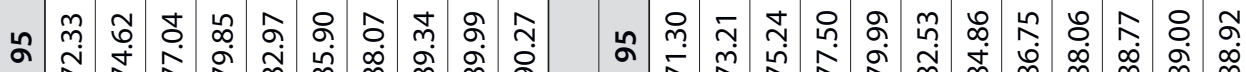

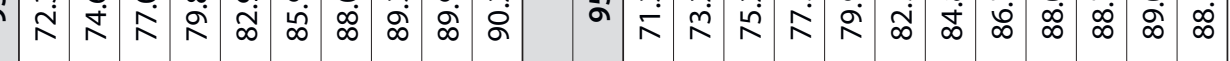

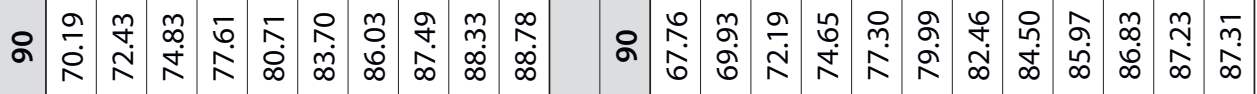

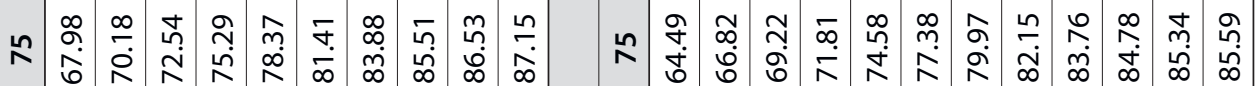

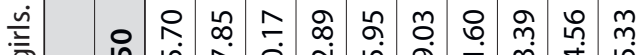

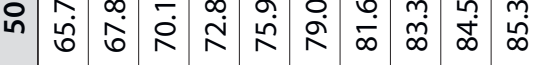

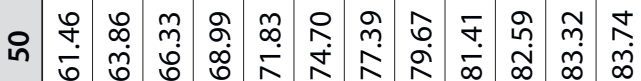

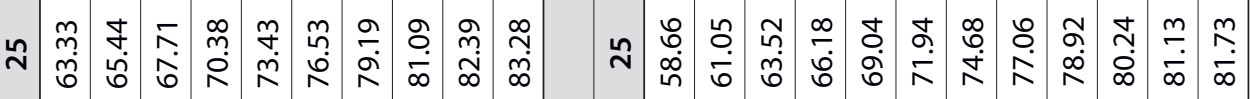

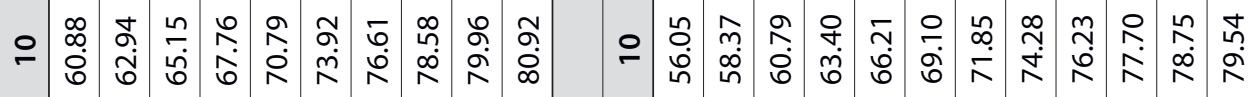

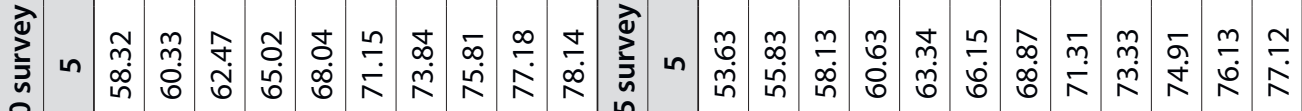

m $\begin{aligned} & t \\ & 0\end{aligned}$ F $m$ 岗

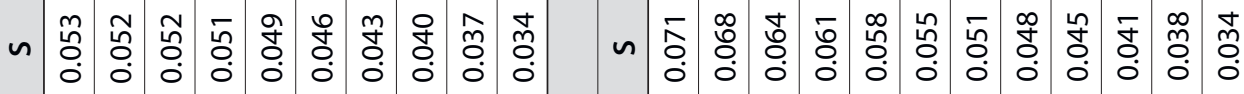

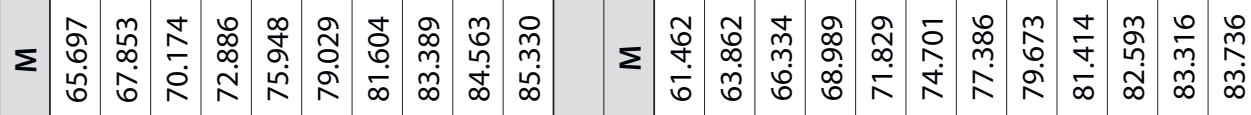

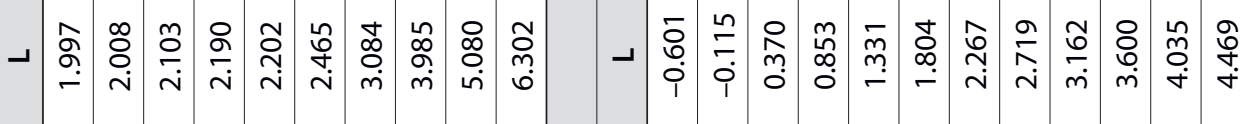

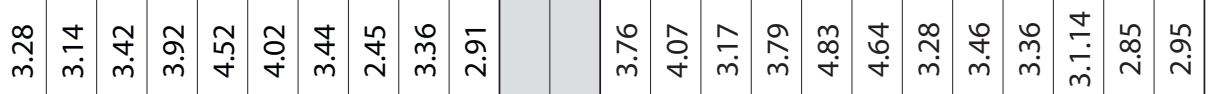
J $=0$

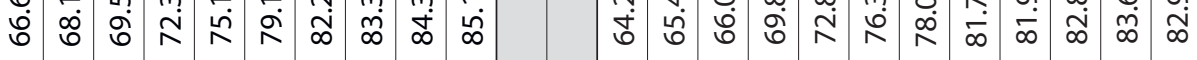

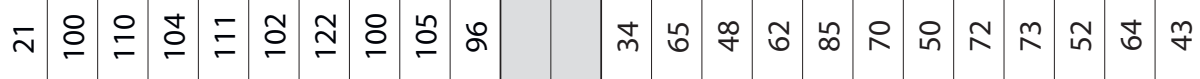

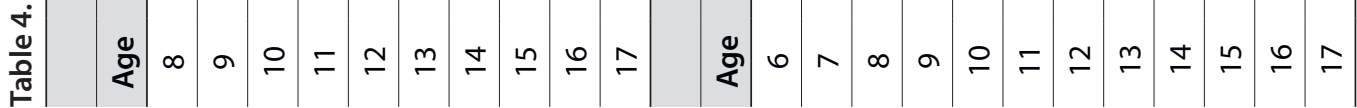




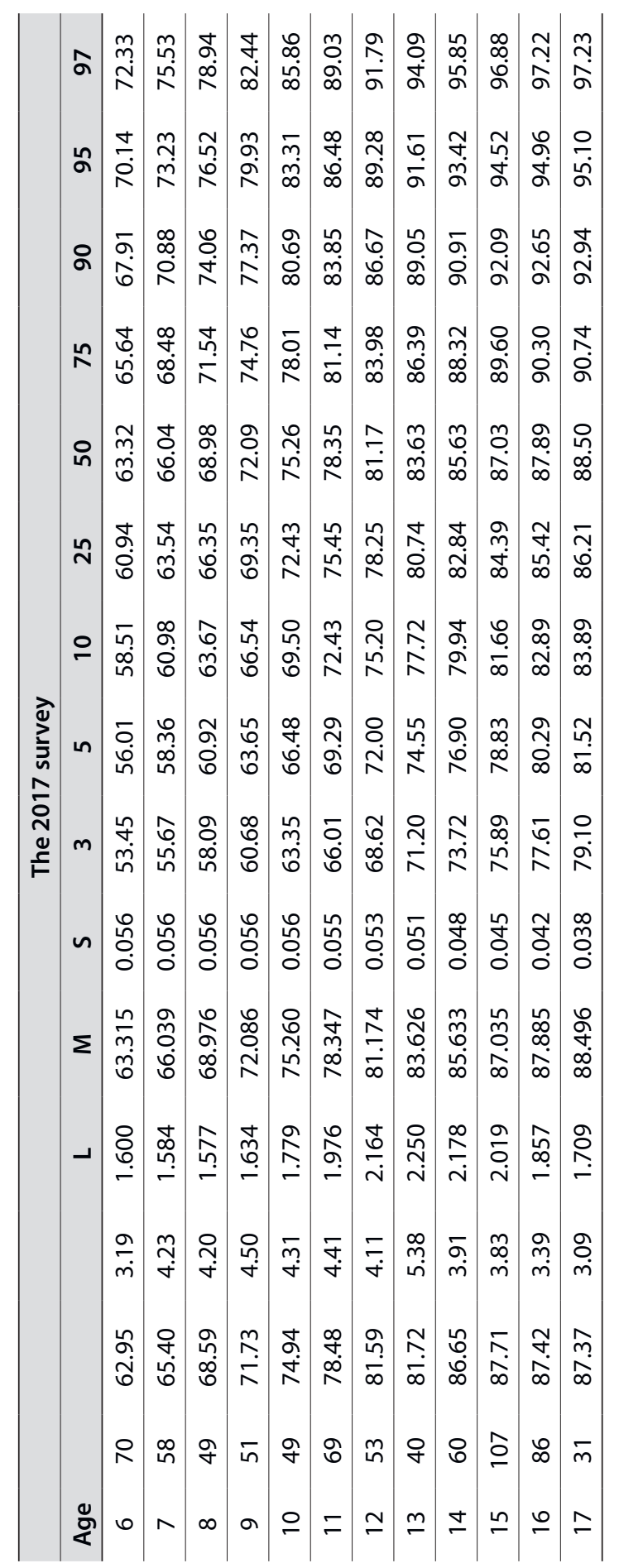




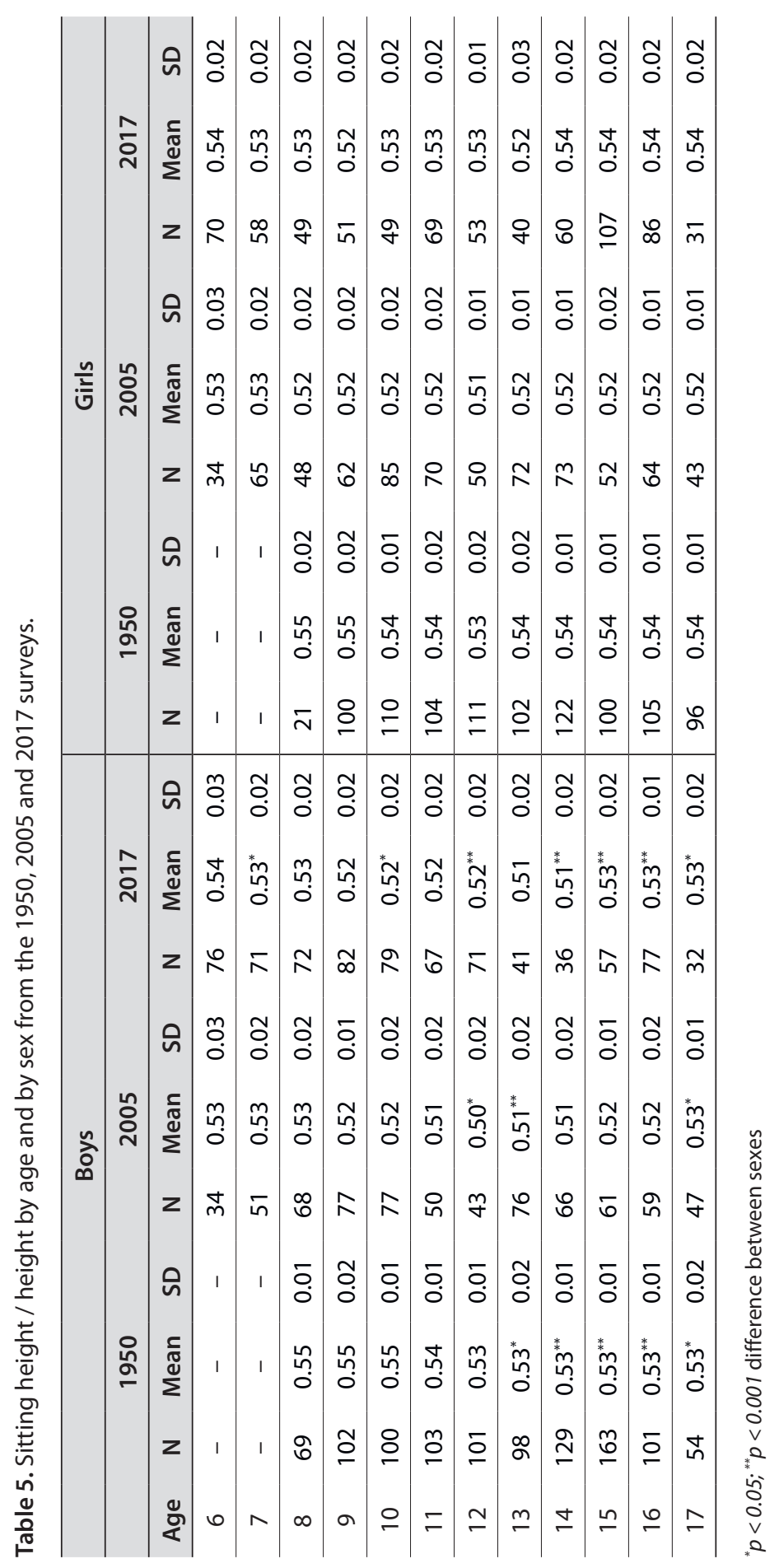


In these three surveys the mean value for sitting height / height ratio was 55\% for boys and $52 \%$ for girls at their respective pre-pubertal ages; however, this value decreased once both sexes reached pubertal ages (Table 5). This decrease of sitting height / height ratio indicates the early onset of a growth spurt and exceeded growth in leg length during respective pubertal ages.

The results of the study showed that the secular increase rate of the height of boys was approximately $2.5 \mathrm{~cm} /$ decade for ages $12,13,14$, and 15 . Between the 1950 and 2017 surveys, the difference in mean height was the largest at age 15 , followed by ages 12,13 and 14 . The height difference in girls between the 1950 and 2005 surveys was found to have the greatest increment at age 11, followed by ages 10,12 and 9 . The increase rate in girls exceeded $2 \mathrm{~cm} /$ decade $(2.21 \mathrm{~cm} /$ decade $)$ only at age 11 , whereas at ages 9,10 and 12 , the increase was approximately $1.9 \mathrm{~cm} /$ decade.

\section{DISCUSSION}

Children's physical growth can be assessed by comparing their body height and other anthropometric measurements [17]. The results of the present study suggest that there has been a significant increase in the mean stature of children since 1950 and that younger generations are taller amongst all the age groups. Sitting height showed a similar tendency with a prominent increase for both sexes. Different timing and intensity of change in linear body segments during the growth period shows sex-specific patterns and results in prominent sexual dimorphism [43]. In addition to the difference in means at the respective ages, the secular increment rate was also found to be different between sexes. The response to environmental improvements as well as deterioration is more marked in males than in females [34]. The results of the present study showed that the final height difference between sexes has been significantly increasing in each consecutive survey year. The difference in final height has doubled for the Turkish population since 1950. When linear growth is almost completed in late adolescence, considerably similar inter-population sexual dimorphism can be observed: $12.8 \mathrm{~cm}$ in Croatian and German populations, $13.0 \mathrm{~cm}$ in Spanish and Hungarian populations, $13.4 \mathrm{~cm}$ in Swiss and Dutch populations, and 13.3 $\mathrm{cm}$ in Czech populations [43].

The effects of quality of life and environmental factors on human growth enable us to hypothesize higher plasticity of leg length relative to sitting height, and this secular trend of increased body height due to the increase in leg length is also associated with better nutrition and health conditions $[5,16,43]$. The 
present results indicate a different pattern in secular changes of height, sitting height and sitting height / height ratio. It has been reported that, in Japan, height increased solely due to an increase in leg length rather than in sitting height, although the proportions of the recent Japanese population differ from those of the European population of the same height [25]. Bodzsar [3] reported that, in the last decades, there has been no change in the growth pattern of sitting height in both sexes of the Hungarian population. We might conclude that the recent secular increment in the height of Ankara children may have been caused by an increment in sitting height as well as leg length. The same tendency has been reported in various countries. Vercauteren et al. [41] reported the secular increase in stature and leg length in Belgian boys between 1960 and 1980, and the secular trend was found to be positive for both traits. The study also emphasizes that, in the Belgian population, the secular increase in stature in the aforementioned period was almost entirely caused by an increase in leg length. The sitting height / height ratio was evaluated in well-to-do İstanbul children aged between 6 and 18 years [9]. The data were collected between 1989 and 2002 [30], and present study results showed similar sitting height / height growth patterns in Istanbul children.

In Europe, major progression in body height was observed predominantly in the first part of the $20^{\text {th }}$ century. Krawczynski et al. [26] report secular changes in body height in children and adolescents in Poland between 1880 and 2000. The most significant differences in body height in the $20^{\text {th }}$ century related to the growth spurt were about $17.1 \mathrm{~cm}$ (12-year-old) and $12.7 \mathrm{~cm}$ (13-year-old) for boys and girls, respectively. In addition, the magnitudes of secular change have not been stable. A period of deceleration has been documented in the 1940s (1946-50). However, the results of the study confirm the intensive process of acceleration of physical development, indicating a slowdown or deceleration thereafter, as reported in some European countries. A similar tendency has been documented in the Netherlands [36] and Belgium in the 1960s and the 1970s [40] and in Sweden in the 1950s and the 1960s [11]. In the USA, Australia [27], Croatia and the Czech Republic, this tendency was observed during the 1980s [26]. Between 1951 and 2001, the mean height of 13-year-old boys and girls increased by $10 \mathrm{~cm}$ and $8 \mathrm{~cm}$ respectively in the Czech Republic. The gradual increase over two centuries has been described as an overall improvement in the standards of living, where the increase in height, the shift in the onset of accelerated growth, and the maximum instantaneous growth rate resulted in logical consequences [42]. Classic examples of secular trends in terms of somatic growth are provided by the London County Council's series of 
studies of height and weight for the first six decades of the $20^{\text {th }}$ century: 1908 , $1938,1949,1954$, and 1966 [10]. In the second half of the $20^{\text {th }}$ century, the secular increase rate was found to be slower in Turkish children. The acceleration in Turkish children is still continuing, similarly to children in China and Portugal [26]. In 1950, the difference in final height between sexes was noted to be $7 \mathrm{~cm}$; however, at present, the difference is $14 \mathrm{~cm}$. When compared to the developed countries that experienced a high degree of secular increase, Turkish children have exhibited a slow rate of secular increase during the second half of the last century.

A review of the present datasets demonstrated positive secular changes in height and sitting height. The datasets presented sexual differences at most ages due to different responses to environmental and socio-economic conditions. In conclusion, remarkable secular increase during the second half of the last century was accelerated by positive changes in the living conditions. This increase was not comparable with the increase experienced in the developed countries due to the economic and political fluctuations. However, further secular increase can be expected in the near future.

\section{ACKNOWLEDGEMENTS}

We would like to thank all the children who voluntarily participated in our surveys as well as the principals and teachers for their collaboration. We are also grateful to Professor Enver Yaşar Bostancı (1923-1995), Ankara University, Anthropology Department, for his pioneering work on auxology and for leading pathways for us to investigate the growth patterns of Ankara children.

\section{FUNDING}

The 2017 Ankara Survey was supported by the Scientific Research Projects Unit of Ankara University [grant number: 16B0649002, 2017].

\section{REFERENCES}

1. Alantar İ.H. (1938). Türk çocuklarında antropometrik ölçüler, Birinci Türk Çocuk Hekimliği Kongre Kitabı, Ekspres Basımevi, 3-31, Ankara.

2. Arcaleni E. (2006). Secular trend and regional differences in the stature of Italians, 1854-1980. Economics and Human Biology, 4(1), 24-38. https://doi. org/10.1016/j.ehb.2005.06.003 
3. Bodzsar E. (1998). Secular Growth Changes in Hungary. In Bodzsar B.E. and Susanne C. (eds.) Secular Growth Changes in Europe. Eotvos Univ. Press, Budapest, 175-205.

4. Bogin B. (1999). Patterns of human growth. Cambridge: Cambridge University Press.

5. Bogin B., Kapell M., Silva M.I.V., Orden A.B., Smith P.K., Loucky J. (2001). How Genetic Are Human Body Proportions? In: Dasgupta P., Hauspie R. (eds) Perspectives in Human Growth, Development and Maturation. Dordrecht: Springer, 205-221. https://doi.org/10.1007/978-94-015-9801-9_17

6. Bostanc1 E.Y. (1954). A research on the growth of stature of Turkish schoolchildren in Ankara. Ankara Universitesi Dil ve Tarih-Cografya Fakultesi Dergisi, 12(1-2), 41-75.

7. Bostanc1 E.Y. (1955). Growth research on trunk and legs in Turkish school children in Ankara. Ankara Üniversitesi Dil ve Tarih-Coğrafya Fakültesi Dergisi, 13(1-2), 70-136.

8. Bostanc1 E.Y. (1956). Growth research on arm, upper arm and forearm length and hand length and width and the correlations in Turkish boys and girls, Ankara Üniversitesi Dil ve Tarih-Coğrafya Fakültesi Dergisi, 14(1-2), 103-203.

9. Bundak R., Baş F., Furman A., Günöz H., Darendeliler F., Saka N., Poyrazoğlu Ş., Neyzi O. (2014). Sitting height and sitting height/height ratio references for Turkish children. Eur J Pediatr, 173(7), 861-869. https://doi.org/10.1007/ s00431-013-2212-3

10. Cameron N. (1979). The growth of London schoolchildren 1904-1966: An analysis of secular trend and intra-county variation. Annals of Human Biology, 6(6), 505-525. https://doi.org/10.1080/03014467900003921

11. Cernerud L., Lindgren G.W. (1991). Secular changes in height and weight of Stockholm schoolchildren born in 1933, 1943, 1953 and 1963. Annals of Human Biology, 18(6), 497-505. https://doi.org/10.1080/03014469100001802

12. Cole T.J., Green P.J. (1992). Smoothing reference centile curves: the LMS method and penalized likelihood. Statistics in Medicine, 11, 1305-1319. https:// doi.org/10.1002/sim.4780111005

13. Cole T.J. (2002). Growth references and standards. In: Cameron N. (ed.), Human growth and development. St Louis: Academic Press, 383-414. https:// doi.org/10.1016/B978-012156651-7/50019-8

14. Çınar N. (1939). Ankara, Devrim ilkokul talebelerinden 433 kız ve erkek çocuk üzerinde antropometrik bir tetkik ve neticeleri, Türk Antropoloji Mecmuası, 15(19-22), 67-79.

15. Duyar İ. (1992). Growth standards in 12-17 years aged Turkish children. Thesis, Ankara: Hacettepe University, Turkey.

16. Eveleth P.B., Tanner J.M. (1990). World Variation in Human Growth (2nd ed.). Cambridge: Cambridge University Press. 
17. Fredriks A.M., van Bruuen S., van Heel W.J.M., Dijkman-Neerincx R.H.M., Verloove-Vanhorick S.P., Wit J.M. (2004). Nation-wide references for sitting height, leg length, and sitting height/height ratio, and their diagnostic values for disproportionate growth disorders. In: Fredriks A.M. (ed.) Growth diagrams 1997. Fourth Dutch Nation-wide Survey. Leiden University, 39-51.

18. Gökçül N. (1939). Ankara İsmetpaşa ilkokul talebelerinden $422 \mathrm{k} 1 \mathrm{z}$ ve erkek Türk çocuğu üzerinde antropometrik araştırmalar ve neticeleri. Türk Antropoloji Mecmuasi, 15(19-22), 176-186.

19. Kansu S.A. (1939). Kiz ve erkek Turk cocuklari uzerinde antropometrik arastirmalar. Belleten, 9, 69-79.

20. Kınay M. (1939). Ankara Gedikli ortaokulu talebelerinden 200 erkek çocuk üzerinde antropometrik bir tetkik ve neticeleri. Türk Antropoloji Mecmuası, 19-22, 176-188.

21. Koca Özer B. (2007a). Somatometric study on the secular change of Turkish population, Thesis: Kyoto, Kyoto University, Japan.

22. Koca Özer B. (2007b). Secular Changes in Height and Leg Length Among Turkish Children During the Last Century. Ankara Üniversitesi Dil ve TarihCoğrafya Fakültesi Dergisi, 47(1), 95-113.

23. Koca Özer B. (2007c). Secular trend in the height and weight of Turkish adults, Anthropological Science, 116(3), 191-199.

24. Komlos J., Baur M. (2004). From the tallest to (one of) the fattest: the enigmatic fate of the American population in the 20th century. Economics and Human Biology, 2(1), 57-74. https://doi.org/10.1016/j.ehb.2003.12.006

25. Kouchi M. (1996). Secular change and socioeconomic difference in height in Japan. Anthropological Science, 104(4), 325-340. https://doi.org/10.1537/ ase. 104.325

26. Krawczynski M., Walkowiak J., Kryzaniak A. (2003). Secular changes in body height and weight in children and adolescents in Poznan, Poland, between 1880 and 2000. Acta Paediatrica, 92(3), 277-282. https://doi. org/10.1111/j.1651-2227.2003.tb00546.x

27. Loesch D.Z., Stokes K., Huggins R.M. (2000). Secular trend in body height and weight of Australian children and adolescents. American Journal of Physical Anthropology, 111(4), 545-556. https://doi.org/10.1002/ (SICI)1096-8644(200004)111:4\%3C545::AID-AJPA9\%3E3.0.CO;2-X

28. Lohman T.G., Roche A.F., Martorell R. (1988). Anthropometric Standardization Reference Manual. Illinois: Human Kinetics Books.

29. Martin R. (1928). Lehrbuch der Anthropologie, Vol. 1. Jena: Verlag von Gustav Fisher.

30. Neyzi O., Furman A., Bundak R., Günöz H., Darendeliler F., Baş F. (2006). Growth references for Turkish children aged 6 to 18 years, Acta Pediatrica, 95, 1635-1641. https://doi.org/10.1080/08035250600652013 
31. Roche A., Sun S. (2003). Human Growth: Assessment and Interpretation. Cambridge: Cambridge University Press.

32. Saatçioğlu A. (1988). Growth standards for 7 to 11-year-old Turkish children. Kuopio: Publications of the University of Kuopio.

33. Schell L.M., Knutsen K.L. (2002). Environmental effects on growth. In: Cameron N. (ed.) Human growth and development, St Louis: Academic Press, 165-195. https://doi.org/10.1016/B978-012156651-7/50009-5

34. Susanne C., Bodzar E.B. (1998). Patterns of secular change of growth and development. In: Bodzar B.E., Susanne C. (eds.) Secular Growth Changes in Europe, Budapest: Eotvos Univ. Press, 5-26.

35. Tanner J.M. (1992). Growth as a measure of nutritional and hygienic status of a population. Hormone Research, 38 (Suppl. 1), 106-115. https://doi. org/10.1159/000182580

36. Tümay S.B. (1938). Çocuklarda büyüme nispetleri. Birinci Türk Çocuk Hekimliği Kongre Kitabı, Ekspres Basımevi, Ankara.

37. Ulijaszek S.J. (1998). The secular trend. In: Ulijaszek S.J., F.E. Johnston, M.A. Preece (eds.) Cambridge Encyclopedia of Human Growth and Development. Cambridge: Cambridge University Press.

38. Weiner J.E.S., Lourie J.A. (1969). Human Biology: A Guide to Field Methods. IBP Handbook, No: 9, Oxford: Blackwell.

39. van Wieringen J.C. (1986). Secular growth changes. Falkner F., Tanner J.M. (eds.) Human Growth: A Comprehensive Treatise, Vol. 3. (2nd ed.) New York: Plenum Press.

40. Vercauteren M., Susanne C. (1985). The secular trend of height and menarche in Belgium: are there any signs of a future stop? European Journal of Pediatrics, 144(4), 306-309. https://doi.org/10.1007/BF00441769

41. Vercauteren M., Hauspie R.C., Susanne C. (1998). Biometry of Belgian boys and girls: change since Quetelet. In: Bodzsar B.E., Susanne C. (eds.) Secular Growth Changes in Europe. Budapest: Eotvos Univ. Press.

42. Vignerova J., Brabec M., Blaha P. (2006). Two centuries of growth among Czech children and youth. Economics and Human Biology, 4(2), 237-252. https://doi. org/10.1016/j.ehb.2005.09.002

43. Zivicnjak M., Naranciæ N.S., Szirovicza L., Franke D., Hrenoviæ J., Bisof V. (2003). Gender-specific growth patterns for stature, sitting height and limbs length in Croatian children and youth ( 3 to 18 years of age). Collegium Anthropologicum, 27(1), 321-334. 


\section{Address for correspondence:}

Başak Koca Özer

Ankara University, DTCF, Department of Anthropology, 06100 Sihhiye Ankara, Turkey.

E-mail: bkozer@ankara.edu.tr

\section{Ayşegül Özdemir}

Ankara University, DTCF, Department of Anthropology, 06100 Sihhiye Ankara, Turkey.

E-mail: ozdemira@ankara.edu.tr 